\title{
Reflection electron energy loss spectroscopy during initial stages of Ge growth on Si by molecular beam epitaxy
}

\author{
Harry A. Atwater \\ Thomas J. Watson Laboratory of Applied Physics, California Institute of Technology, Pasadena, \\ California 91125 \\ Channing C. Ahn \\ W. M. Keck Laboratory of Engineering Materials, California Institute of Technology, Pasadena, \\ California 91125
}

(Received 10 July 1990; accepted for publication 23 October 1990)

\begin{abstract}
Using a conventional reflection high-energy electron diffraction gun together with an electron energy loss spectrometer, we have combined in situ measurements of inelastic scattering intensities from Si $L_{2,3}$ and $\mathrm{Ge} L_{2,3}$ core losses with reflection electron diffraction data in order to analyze the initial stages of Ge heteroepitaxy on $\mathrm{Si}(001)$. Diffraction data indicate an initial layer-by-layer growth mode followed by island formation for Ge thicknesses greater than $0.8-1.1 \mathrm{~nm}$. The electron energy core loss data are consistent with a simple model of grazing incidence electron scattering from the growing Ge film. Reflection electron energy loss spectroscopy is found to be highly surface sensitive, and the energy resolution and data rate are also sufficiently high to suggest that reflection electron energy loss spectroscopy may be a useful real-time, in situ surface chemical probe during growth by molecular beam epitaxy.
\end{abstract}

The precise dimensional control and low growth temperatures of modern epitaxial growth techniques have made it possible to tailor compositionally modulated thin films on an atomic level. A significant factor currently limiting far greater insight about and control of epitaxial growth is a relative lack of in situ chemical analysis techniques which are compatible with the requirements of typical growth environments, such as long working distance and compatibility with deposition sources. The most widely employed in situ probe for molecular beam epitaxy, reflection high-energy electron diffraction (RHEED), is sensitive to surface crystallographic structure and morphology, but does not provide direct measurements of surface composition. Several approaches have been taken to surface compositional analysis during growth, such as reflection mass spectrometry ${ }^{1}$ and ellipsometry. ${ }^{2}$ Each of these techniques is an indirect indication of surface composition. Another approach is reflection electron energy loss spectrometry, which is a direct probe of surface composition, since electrons inelastically scattered from the surface are spectroscopically analyzed.

Electron energy loss spectrometry in the transmission electron microscope has become an important analytic tool complementary to imaging and diffraction and has typically been done in transmission geometry. ${ }^{3}$ Moreover, analysis of extended electron loss fine structure has made possible investigations of local order in solids which were previously confined to intense $x$-ray source facilities, such as a synchrotron. ${ }^{4}$ Experiments conducted in a transmission electron microscope have demonstrated that electron energy loss spectrometry is also possible in reflection mode. ${ }^{5,6}$ Other experiments using a low-energy electron source in a surface analysis system, demonstrated the use of electron energy loss spectroscopy in the analysis of $\mathrm{Cu}$ growth on $\mathrm{Ag}(111))^{7}$ In this letter, we introduce the use of reflection electron energy loss spectrometry (REELS) as an in situ technique for analysis of crystal growth in a conventional molecular beam epitaxy system using a RHEED electron beam source, and discuss application to Ge heteroepitaxy on Si.

The scattering configuration employed for REELS in the present work is similar to a conventional RHEED configuration, with an electron beam incidence angle of $\phi=37$ mrad. Structural analysis by RHEED at $30 \mathrm{keV}$ with an emission current of $30 \mu \mathrm{A}$ was performed simultaneously with the REELS measurements. The RHEED screen was viewed in reflection, and a $3 \mathrm{~mm}$ aperture in the RHEED screen formed the entrance to the electron energy loss spectrometer. The spectrometer is a Gatan 607 second-order corrected sector identical to that normally used in a transmission electron microscope. The object point of the spectrometer is the sample itself, located $\sim 33 \mathrm{~cm}$ from the spectrometer entrance, giving a collection semiangle of 5 mrad. The spectrometer energy resolution, calculated at 25 $\mathrm{keV}$ using the first-order matrix coefficients ${ }^{8}$ for this sector and an assumed $90 \mu \mathrm{m}$ beam waist, is $\sim 5 \mathrm{eV}$. Assuming an incident thermal beam spread of $1.5 \mathrm{eV}$, and high voltage power supply resolution of $3 \mathrm{eV}$, the energy resolution of the system is expected to be $\sim 6 \mathrm{eV}$. Experimental measurements of the energy width of the through beam (i.e., the beam which does not strike the sample) indicated a system resolution of $\sim 7 \mathrm{eV}$. While this is not a highenergy resolution, it is more than adequate for quantitative analysis of core losses.

Initial experiments concentrated on characterizing the optimum scattering geometry for observation of core loss edge intensities for $\mathrm{Si}$ and Ge substrates. Several diffraction conditions were investigated: (i) specular reflection coincident with a Bragg peak of the substrate (in-phase), (ii) specular reflection not coincident with a substrate Bragg peak (out-of-phase), and (iii) a surface resonance condition, where the out-of-phase specular reflection intensity 


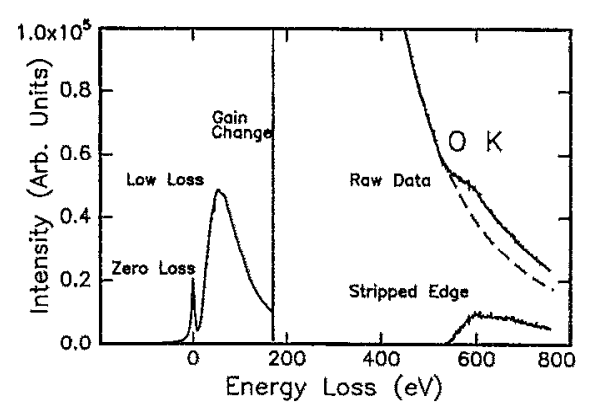

FIG. 1. REELS spectrum illustrating $\mathrm{O} K$ edge from $\simeq 3 \mathrm{~nm}$ native $\mathrm{SiO}_{2}$ on $\mathrm{Si}(001)$ after sample insertion, and before $\mathrm{SiO}_{2}$ desorption by heating.

was enhanced by coincidence of an oblique Kikuchi line with the specular beam spot. ${ }^{9,10}$ For Ge substrates, the Ge $L_{2,3}$ edge was observable in all three conditions, however better ratios of edge intensity to inelastic background intensity were obtained for conditions (ii) and (iii). It has been reported by Wang et al. that condition (iii) scattering, corresponding to surface resonance, is required to obtain acceptable ratios of core loss intensity to inelastic background intensity. ${ }^{6}$ Little difference was observed in the ratio of edge intensity to inelastic background between conditions (ii) and (iii) above in the present work. However, it was determined qualitatively that surface sensitivity is strongly enhanced by adoption of surface resonant scattering conditions. For example, after growth of $0.3-0.5 \mathrm{~nm}$ of $\mathrm{Si}$ on a $\mathrm{Ge}(001)$ substrate, the Ge $L_{2,3}$ core loss intensity from the substrate was not observable. For nonresonant conditions, the substrate core loss intensity is still observable after growth of a $2.0-3.0 \mathrm{~nm}$ thick overlayer, as discussed below.

Films were grown in a custom-designed molecular beam epitaxy system, which is equipped with electron beam sources for deposition of $\mathrm{Si}$ and $\mathrm{Ge}$. Although our growth chamber normally achieves base pressures of $1 \times 10^{-10}$ Torr, addition of the electron energy loss spectrometer, which is not of ultrahigh vacuum design in its current configuration, resulted in base pressures of $5 \times 10^{-9}$ Torr with pressure rising to $1-2 \times 10^{-8}$ Torr during deposition. The $\mathrm{Si}$ buffer layers and Ge films were epitaxial, but transmission electron microscopy indicated stacking faults in the Si buffer layers for some samples.

After insertion into the growth chamber, Si (001) substrates were coated with a thin native $\mathrm{SiO}_{2}$ layer, judged to be $\leqslant 3 \mathrm{~nm}$ thick since this layer was desorbed prior to $\mathrm{Si}$ buffer layer growth by briefly heating the substrate to $800^{\circ} \mathrm{C}$. Before desorption, the RHEED pattern consisted of broad, strongly modulated streaks, and no surface reconstruction was visible. In these conditions, the $O K$ edge is clearly visible in the reflection electron energy loss spectrum, as shown in Fig. 1.

Following growth of a $300 \mathrm{~nm}$ Si buffer layer on a $\mathrm{Si}$ (001) substrate, a streaked RHEED pattern with the $\mathrm{Si}$ $100-(2 \times 1)$ reconstruction characteristic of clean surfaces was observed. Ge was grown at a rate of $0.84 \mathrm{~nm} / \mathrm{min}$ on (001) $\mathrm{Si}$ substrates at a temperature of $410^{\circ} \mathrm{C}$. Film thickness was measured using quartz crystal sensors which had
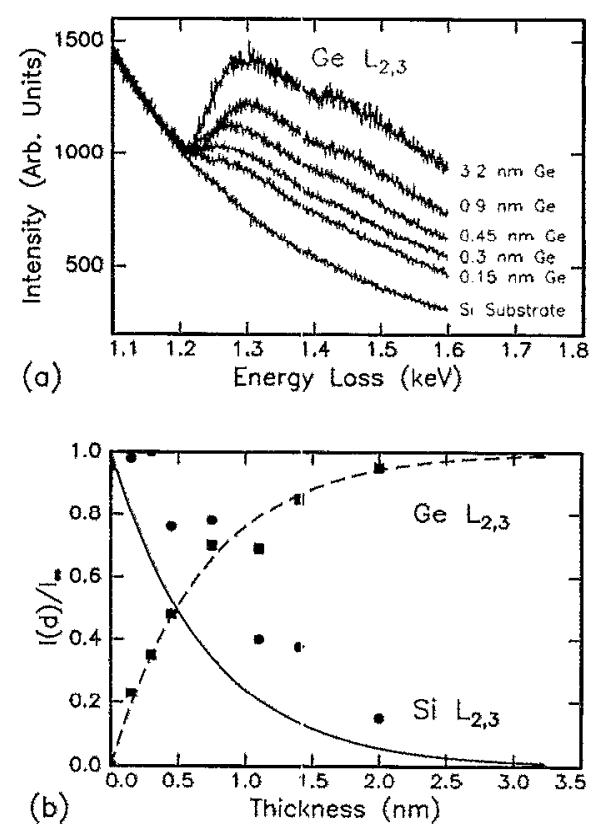

FIG. 2. REELS spectra illustrating change in Ge $L_{2,3}$ edge intensity with Ge thickness shown in (a); variation of normalized $\mathrm{Ge} L_{2.3}$ and $\mathrm{Si} L_{2.3}$ intensities with coverage shown in (b).

been previously calibrated using Rutherford backscattering spectrometry. Growth was briefly interrupted to collect electron energy loss spectra. Energy loss spectra taken in scattering condition (ii) are shown in Fig. 2(a) as a function of Ge thickness. The collection time for each spectrum was $40 \mathrm{~s}$. The appearance of the Ge $L_{2,3}$ edge is clearly visible in the electron energy loss spectrum at Ge thicknesses as small as $0.15 \mathrm{~nm}$. For Ge thicknesses $d \geqslant 0.3 \mathrm{~nm}$, the $\mathrm{Ge} L_{1}$ edge is also visible. It should be emphasized that the results in Fig. 2(a) are data from single spectra, and have not been averaged or otherwise processed, except to normalize the pre-edge background intensity. We expect the pre-edge intensity to remain constant, since the scattering geometry was unchanged during film growth. The variation of the core less intensities with nominal Ge film thickness for the $\mathrm{Ge} L_{2,3}$ edge (film) and the Si $L_{2,3}$ edge (substrate) is shown in Fig. 2(b).

Also for $d \geqslant 0.3-0.4 \mathrm{~nm}$, the RHEED pattern consisted of sharp spots characteristic of diffraction from a rough surface. The change in the RHEED pattern was interpreted as marking the onset of islanding, which is consistent with other observations of Ge heteroepitaxy on Si that indicate a Stranski-Krastanov growth mode. ${ }^{11,12}$ This conclusion was checked by ex situ reflection electron microscopy and cross-sectional transmission electron microscopy, performed in a Phillps EM-430 microscope. Figure 3 is a transmission electron micrograph taken along the [011] zone axis of $\mathrm{Ge}$ islands on $\mathrm{Si}(001)$ for a sample with a nominal coverage of $d=3.2 \mathrm{~nm} \mathrm{Ge}$ on Si. For this sample, Ge islands were approximately $15-30 \mathrm{~nm}$ in diameter, with an average interisland distance of $\sim 50-100 \mathrm{~nm}$. The island contact angle on the substrate was $\sim 65^{\circ}$.

The normalized $\mathrm{Ge} L_{2,3}$ and $\mathrm{Si} L_{2,3}$ intensities shown in Fig. 2 were determined from power law background fits for 


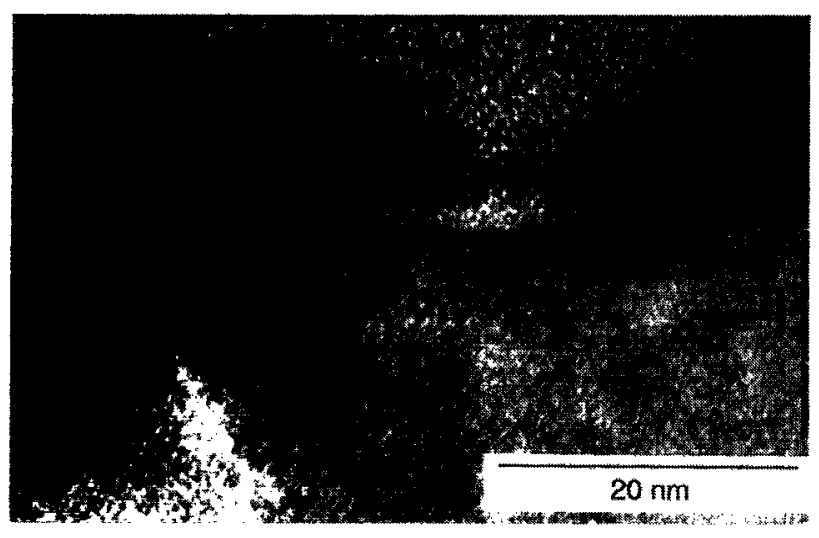

FIG. 3. High-resolution cross-sectional electron micrograph of Ge islands on $\mathrm{Si}(001)$ for a sample with nominal thickness of $3.2 \mathrm{~nm}$ Ge grown at $T=410^{\circ} \mathrm{C}$

$200 \mathrm{eV}$ windows. These intensities vary gradually and in a complementary manner with coverage in the range $0<d<3.2 \mathrm{~nm}$. For nonresonant conditions, it is possible to interpret the changes in intensities with coverage as being due to electron energy loss at grazing incidence angle $\phi$ from thin uniform overlayer of thickness $d$, which is in most respects very similar to transmission electron energy loss, with a core loss scattering yield $I_{c}$ from an elemental overlayer given by

$$
I_{c}=I(\Delta E) \sigma(\Omega, \Delta E) N_{\mathrm{el}}\left[\lambda\left(1-e^{-d / \sin \phi \lambda}\right)\right],
$$

where $I(\Delta E)$ is the integrated low-loss intensity, and $N_{\mathrm{el}}$ is the elemental concentration of a given species in the overlayer. The integrated elemental scattering cross section, $\sigma(\Omega, \Delta E)$ is dependent on the collection semiangle $\Omega$, and the energy loss window for data collection $\Delta E$. The effective thickness for electron scattering is equal to the projected overlayer thickness $d / \sin \phi$ for very thin overlayers of uniform thickness. In thicker overlayers, the effective thickness is limited by $\lambda$, the mean free path for total electron scattering. The solid line and dashed line in Fig. 2(b) are the intensities predicted using this simple transmission model for $\mathrm{Si}$ and $\mathrm{Ge}$, respectively, assuming a uniform film thickness, and an electron mean free path at an energy of $30 \mathrm{keV}$ of $\lambda \simeq 20 \mathrm{~nm} .{ }^{13}$ Excellent agreement is obtained between the theoretical and experimental intensities for the Ge $L_{2,3}$ edge. The agreement between theoretical and experimental intensities for the Si $L_{2,3}$ edge is not as good, possibly due to multiple inelastic scattering in the low-loss region, resulting in less accurate background fits. At an incidence angle of $\phi=37 \mathrm{mrad}$, the actual film thickness at which the projected thickness is equal to the total mean free path is $d=0.74 \mathrm{~nm}$. Since Ge growth on Si results in island formation, a more refined analysis could be devel- oped which accounts for the geometrical differences in scattering from an overlayer of uniform thickness and one consisting of islands. However, for the parameters of this experiment (e.g., island density, island contact angle, electron mean free path, beam incidence angle), these geometrical differences are expected to be minor.

In conclusion, we have introduced reflection electron energy loss spectroscopy as an in situ probe of surface composition during molecular beam epitaxy, and have applied the technique to the study of Ge growth on Si (001). Core loss intensities are observable in thin overlayers for surface resonant as well as nonresonant conditions. The changes in relative intensities of $\mathrm{Ge} L_{2,3}$ and $\mathrm{Si} L_{2,3}$ core losses from a Ge overlayer on a Si substrate are consistent with a simple model of grazing incidence electron scattering. Considerable work remains to be done in assessing the resonance enhancement of surface sensitivity, as well as absolute and relative chemical sensitivity in order to develop reflection electron energy loss spectroscopy for more general growth conditions (e.g., growth of compound materials). Considerable gains in data rate for deep core losses could also be realized by use of an electron energy loss spectrometer with parallel detection. Nonetheless, these initial experiments have demonstrated that the surface sensitivity, data rate, and energy resolution are sufficiently high to suggest that reflection electron energy loss spectroscopy has great potential as a practical real time, in situ probe of surface composition during epitaxial growth.

This work was supported by the National Science Foundation P.Y.I.A. program (DMR-8958070) and the Materials Research Group program (DMR-8811795). We would like to acknowledge helpful discussions with P. Rez, encouragement from $M$. De Crescenzi and J. Dinan, and experimental assistance by C-J. Tsai. We thank Philips Electronic Instruments for the loan of the spectrometer.

' J. Y. Tsao, T. M. Brennan, and B. E. Hammons, Appl. Phys. Lett. 53, 288 (1988)

${ }^{2}$ D. E. Aspnes, W. E. Quinn, and S. Gregory, Appl. Phys. Lett. 56, 2569 (1990).

${ }^{3}$ R. F. Egerton, Electron Energy Loss Spectroscopy in the Electron Microscope (Plenum, New York, 1986)

${ }^{4}$ M. M. Disko, G. Meitzner, C. Ahn, and O. L. Krivanek, J. Appl. Phys. 65, 3295 (1989).

${ }^{5}$ Z. L. Wang and J. M. Cowley, Surf. Sci. 193, 501 (1988).

${ }^{6}$ Z. L. Wang and R. F. Egerton, Surf. Sci. 205, 25 (1988).

${ }^{7}$ T. Tyliszczak, M. De Crescenzi, and A. P. Hitchcock, Phys. Rev. B 37 , 10664 (1988).

${ }^{8}$ K. L. Brown, Adv. Part. Phys. 1, 71 (1967).

${ }^{9}$ E. G. McRae, Rev. Mod. Phys. 51, 541 (1979).

${ }^{10} \mathrm{~S}$. Miyake and K. Hayakawa, Acta. Cryst. A 26, 60 (1970).

${ }^{11}$ M. Zinke-Allmang, L. C. Feldman, S. Nakahara, and B. A. Davidson, Phys. Rev. B 39, 7848 (1989).

${ }^{12}$ M. Krishamurthy, J. S. Drucker, and J. A. Venables, Mater. Res. Soc. Symp. Proc. Vol. 198 (to be published, 1990).

${ }^{13}$ W. Brünger and W. Menz, Zeit. Phys. 184, 271 (1965). 